\title{
LAPURDUTH
}

Euskal ikerketen aldizkaria | Revue d'études basques |

Revista de estudios vascos | Basque studies review

$3 \mid 1998$

Numéro III

\section{A propos des mots basques ahuntz « chèvre » et lepo «cou, col»}

Michel Morvan

\section{OpenEdition}

\section{Journals}

Édition électronique

URL : https://journals.openedition.org/lapurdum/1664

DOI : 10.4000/lapurdum.1664

ISSN : 1965-0655

\section{Éditeur}

IKER

Édition imprimée

Date de publication : 1 octobre 1998

Pagination : 29-32

ISBN : 2-84127-152-8

ISSN : $1273-3830$

Référence électronique

Michel Morvan, «A propos des mots basques ahuntz « chèvre » et lepo « cou, col » », Lapurdum [En ligne], 3 | 1998, mis en ligne le 01 septembre 2010, consulté le 26 février 2022. URL : http:// journals.openedition.org/lapurdum/1664; DOI : https://doi.org/10.4000/lapurdum.1664 


\section{À PROPOS DES MOTS BASQUES AHUNTZ "CHEVRE" ET LEPO "COU, COL"}

\section{Ahuntz "chèvre".}

Voici un terme qui, comme bien d'autres termes basques, aura sans doute fait couler beaucoup d'encre. Mais, en dépit de tous ces efforts louables, rien de définitif n'a pu être établi dans le domaine comparatif.

Je n'ai pas l'intention de faire mieux que mes prédécesseurs, à ceci près que, pour ce qui me concerne, j'ai le courage de choisir la solution qui me paraît la meilleure et de le dire.

Ce n'est pas le cas du prétendu "Dictionnaire étymologique basque" d'A. Tovar et M. Agud' qui, comme on va pouvoir le constater une fois de plus avec l'exemple de ahuntz "chèvre", se contente d'énumérer les multiples hypothèses qui ont été émises en mettant tout sur le même plan, et en reprenant sans rien y changer (y compris même la pagination!) ce qui était seulement à l'origine un ensemble de "matériaux" en vue de l'élaboration d'un futur dictionnaire étymologique ${ }^{2}$.

Noyée dans tout ce "fatras" qui n'est en fait qu'un historique de toutes les hypothèses émises, la meilleure comparaison finit donc par passer inaperçue ou du moins par être mise au même rang que toutes les autres. Je précise que je dis bien "la meilleure comparaison" et non pas obligatoirement la bonne. Mais c'est la meilleure qui doit figurer dans un vrai dictionnaire étymologique jusqu'à ce qu'on trouve éventuellement mieux. Ce nivellement des hypothèses est préjudiciable à une saine démarche scientifique. Nous ne devons pas attendre cent sept ans pour avancer dans les recherches comme le font trop souvent les linguistes conservateurs, tétanisés par une prudence excessive dont les effets sont aussi néfastes que le manque de prudence. Seule la combinaison de la prudence et de la hardiesse, nullement antinomiques en l'occurrence, peut faire avancer la science. Je précise bien que je parle ici des comparaisons du basque avec d'autres langues et non de la reconstruction interne au basque, dans le cas présent ahuntz $<*$ anuntz, qui, elle, me semble au contraire fort pertinente.

Voici la première partie du texte des Materiales... :

«AUNTZ, ahuntz L, BN. S, ahüntz, aintz R "cabra". Sobre la distribución de formas v. Mich. BAP 6, 454. Este reconstruye une forma *anu(n)-tz, (de *anu acaso según Mich. FHV 115 s.) a base del top. Anuncibay (Hom. Urq. 2, 485). Es posible que esta reconstrucción sea conciliable con la teoría de Sch. RIEV 7, 314 que comparaba auntz con antzu (q.u.), antxu "oveja estéril", aunzu AN "estéril" (dicho de animales) ; cf. artantzu "oveja de tres años que no ha parido", artantxu 
"oveja para cebar". Sch. supone que se trataría de derivados del románico anniculus, etc. (Corominas lo cree muy poco probable, pues tropieza con la fonética); $\mathrm{cf}$. bearn. anesque "oveja añoja".

Lafon $B A P$ 6, 307 señala que sobre auntz y a(h)uña se puede reconstruir una raiz *a(u)n-, que podria reducirse considerando la a protética. Sch. 1.c. discute sobre $-t z$, y no resuelve si se trata de un suf. o de un elemento de la raíz : antxu$m e$, an(t)xume admite una doble análisis, pues se hallan igualmente txume y ume, con el mismo significado. Uhl. RIEV 3,421 llega a decir que - $t z$ es femenino comparando con ahuña... (No insiste en Oud. Lagen 358, aunque sí en el carácter sufijal de -tz). Como dice Mich. FHV 88, aguña salac, ahüne S. añe "cabrito" están emparentados con auntz.

Para resolver este complicado problema hay que señalar las formas gasc. ansoülh "cabrito", ansoùlho "cabra joven, añoja", que cita Rolhfs Gascon 40, y que acaso son pervivencias del sustrato. O, como cree Tovar, del vasco. Corominas ve en aun $(t) z$ una palabra paleo-vasca.

El mismo Sch. 1.c. admite que una raiz ${ }^{*} a-n u$ sería comparable con ár. "anaq" "joven hembra de cabra"; pero un originario auntz le parece mejor compararlo con asir. enzu, ár. 'anz, hebr. 'enz, songhai (quizá préstamo ár. ?) hantsi "cabra" (etc.). »

On notera tout d'abord que, comme à leur habitude, les auteurs mettent la forme "sudiste" auntz en entrée au lieu de ahuntz dont le $h$ est étymologique quoi qu'en dise R.L. Trask qui affirme péremptoirement qu'il n'y a pas de $h$ étymologique en basque ${ }^{4}$. Si la reconstruction interne *anuntz est bonne, appuyée sur l'existence du toponyme Anuncibay, le $h$ de ahuntz est forcément étymologique puisque représentant la place laissée par $-n$ - qui s'est affaibli dans le type classique de l'emprunt latin anatem > ahate "canard". Le fait que la nasalisation se soit reportée sur les voyelles ne change rien à l'affaire. De plus des termes comme harri "pierre" prouvent l'existence d'une aspiration étymologique.

Le bon sens nous invite à considérer que l'animal domestiqué qu'est la chèvre semble provenir de la zone de révolution agro-pastorale néolithique qui a eu comme point de départ le Proche-Orient et s'est diffusée progressivement vers l'ouest jusqu'à la pointe extrême de l'Europe occidentale. En conséquence le Bassin méditerranéen est fortement concerné par cette expansion néolithique et les langues des peuples qui l'entourent doivent être examinées. Parmi ces langues, autant chercher de préférence, en premier lieu, du côté de celles qui sont les plus proches du point de vue proche oriental. De même que le nom du vin est issu du substrat méditerranéen (waï en arabe sémitique), on peut constater que dans la liste sans fin des Materiales... une seule forme peut vraiment retenir l'attention: il s'agit de l'arabe 'anz "chèvre". C'est de toute évidence la forme la plus proche du basque *anuntz et c'est celle que je retiendrai jusqu'à plus ample informé pour mon futur dictionnaire étymologique en cours d'élaboration. S'agit-il d'un emprunt ou d'un terme commun issu du vieux substrat proche oriental? Cette seconde solution paraît nettement préférable, car on ne voit pas pourquoi le basque aurait emprunté le mot à l'arabe comme le croyait Löpelmann (EWBS). 


\section{Lepo "cou, col".}

Il y a quelque temps j'avais déjà pensé à une note sur ce terme basque largement utilisé en toponymie, et surtout en oronymie. La forme même du terme m'incita à me poser la question de savoir si par hasard ce mot ne suivrait pas la même formation et les mêmes évolutions que d'autres termes basques tels que leka "gousse" dont on peut trouver une variante teka dans le lexique basque, ce qui permet de lui donner une étymologie, puisqu'il s'agit visiblement d'un emprunt au roman teka dont le représentant le plus connu est le gascon teca "gousse". Le terme provient du latin theca "boîte, étui", et au-delà du grec qui nous a donné tous les mots terminés en -thèque comme bibliothèque, discothèque, pinacothèque, etc. avec le sens de "contenant".

En ce qui concerne lepo "col", il n'y a pas de forme variante tepo dans le lexique basque. J'étais donc sur le point d'abandonner mon hypothèse lorsque mon attention, à l'occasion de recherches sur les montagnes du Pays basque, fut soudain attirée par deux oronymes situés dans une zone très localisée de la HauteNavarre tout près de la frontière, et bien visibles sur la carte de l'IGN n' 1346 ouest (Saint-Etienne-de-Baiggorry), au-delà d'Urepel par conséquent. Sur la frontière se trouve le col de Burdincurutcheta $(1092 \mathrm{~m}$.) près du mont Lindus $(1220 \mathrm{~m}$.). De l'autre côté de la frontière ce col est indiqué très clairement Burdincuruch-tepoa avec $t$ - initial au lieu du $l$-attendu pour lepo. Il ne s'agit certainement pas d'une faute de typographie, car juste un peu plus loin au sud-ouest se trouve toujours du côté navarrais, l'oronyme Sorotepo avec la même graphie tepo pour "col".

Il ne fait donc plus aucun doute pour moi que le terme basque lepo "cou, col" a bel et bien eu une variante tepo selon le même modèle que leka/teka "gousse" mentionné ci-dessus, même si cette variante a disparu du lexique et des dictionnaires basques actuels (et même du lexique médiéval : 1218 lepoa etc.).

La question qui se pose maintenant est de savoir si ce terme est emprunté au roman comme dans le cas de leka < teka "gousse", ou non, ce qui est possible car l'alternance $t / l$ ne joue pas forcément que pour les emprunts. Le réponse est bien difficile à donner. Il existe effectivement un vieux terme substratique *tep- "hauteur" en pré-indoeuropéen reconnu jusqu'en turc (tep, tepe "colline"), en vieil égyptien (tep "tête") et jusqu'en aztèque (nahuatl tepe-tl "montagne"). Dans le même temps les langues romanes possèdent elles aussi un terme tepe, tepa "motte, coteau" (notamment en gascon du Gers, cf. Simin Palay, Dictionnaire du béarnais et du gascon modernes) qui a peut-être son origine dans ce terme substratique pré-indoeuropéen.

\section{Michel MORVAN}

UPRESA 5478 Bordeaux III- CNRS 


\section{NOTES.}

1. A. Tovar, M. Agud, Diccionario etimológico vasco, II, Ardun-Beuden, Saint-Sébastien 1990.

2. A. Tovar, M. Agud, "Materiales para un diccionario etimológico de la lengua vasca", ASIU, 1988 (ASJU XIIL-1, 1989, p. 496-497 pour la présente note).

3. Tovar/Agud, op. cit., p. 496-497

4. R.L. Trask: "Thc pre-basque aspiration was not etymological, but was merely a suprasegmental feature" ; mais il est obligé d'ajouter aussitôt qu'il y a une poignée d'exceptions : "with a tiny handful of possible exceptions" (sic), "Basque and Dene-Caucasian : a critique from the basque side", Mother Tonguc, I, 1995, p.9-10. 